\title{
Superb microvascular imaging technology of ultrasound examinations for the evaluation of tumor vascularity in hepatic hemangiomas
}

\author{
Sun Kyung Jeon ${ }^{1}$, Jae Young Lee ${ }^{1,2}$, Joon Koo Han ${ }^{1,2}$ \\ 'Department of Radiology, Seoul National University Hospital, Seoul National University \\ College of Medicine, Seoul; ${ }^{2}$ Institute of Radiation Medicine, Seoul National University \\ Medical Research Center, Seoul, Korea
}

Purpose: This study aimed to investigate and categorize the diverse features of hepatic hemangiomas on superb microvascular imaging (SMI) in a relatively large prospective study.

Methods: In this prospective study, 70 patients with 92 hepatic hemangiomas were consecutively enrolled. All nodules were radiologically confirmed with the typical imaging features of hepatic hemangiomas on dynamic computed tomography (CT) or magnetic resonance imaging (MRI). Using SMI, all lesions were evaluated and categorized into subgroups according to the flow pattern on SMI. Differences in the frequencies of SMI patterns according to lesion size and enhancement patterns on dynamic CT or MRI were also compared.

Results: In $67.4 \%$ (62/92) of hemangiomas, tumor vascularity was detected using SMI, while $32.6 \%(30 / 92)$ did not show any signal on the SMI examination, and the absence of an SMI signal was not shown in rapidly enhancing hemangiomas (0\% [0/30] vs. 100\% [30/30], $\mathrm{P}=0.002)$ and was more frequent in lesions $<2 \mathrm{~cm}$ than in lesions $\geq 2 \mathrm{~cm}(44.0 \%$ [22/50] vs. $2.7 \%$ [8/42], $P=0.011)$. In hepatic hemangiomas in which vascularity was detected $(n=62)$, the strip rim pattern was the most common SMI pattern of hepatic hemangiomas $(48.4 \%, 30 / 62)$, followed by the nodular rim pattern involving spotty dot-like engorged vessels $(37.1 \%, 23 / 62)$.

Conclusion: The evaluation of the inner vascularity of hepatic hemangiomas with SMI was feasible for most hemangiomas, especially in larger $(\geq 2 \mathrm{~cm})$ or rapidly enhancing hemangiomas. The most frequent SMI patterns of hepatic hemangiomas were the strip rim pattern and nodular rim pattern.

Keywords: Hemangioma; Ultrasonography; Doppler ultrasonography; Microvessels

\section{Introdutcion}

Ultrasonography (US) has been widely used as a first-line imaging modality for the detection of focal liver lesions (FLLS) due to its broad availability and noninvasiveness [1]. Among incidentally

Received: November 8, 2020 Revised: February 10, 2021 Accepted: February 14, 2021

Correspondence to: Jae Young Lee, MD, Department of Radiology, Seoul National University Hospital, Seoul National University College of Medicine, 101 Daehak-ro, Jongno-gu, Seoul 03080, Korea

Tel. +82-2-2072-3073

Fax. +82-2-743-6385

E-mail: leejy4u@snu.ac.kr

This is an Open Access article distributed under the terms of the Creative Commons Attribution NonCommercial License (http://creativecommons.org/ licenses/by-nc/4.0/) which permits unrestricted noncommercial use, distribution, and reproduction in any medium, provided the original work is properly cited.

Copyright (C) 2021 Korean Society of Ultrasound in Medicine (KSUM)

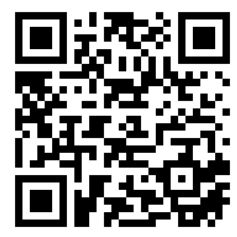

How to cite this article:

Jeon SK, Lee JY, Han JK. Superb microvascular imaging technology of ultrasound examinations for the evaluation of tumor vascularity in hepatic hemangiomas. Ultrasonography. 2021 Oct;40(40):538-545. 
detected FLLs, hepatic hemangiomas are the most common benign liver lesions besides hepatic cysts, and differentiating them from malignant lesions is clinically important, as their treatment and clinical outcomes differ significantly [2]. However, the exact differentiation of hepatic hemangiomas from malignant tumors is often difficult on US, as B-mode US echogenicity and the appearance of hepatic hemangiomas have been known to vary [3].

In this regard, several studies have reported that information on tumor vascularity can be helpful in differentiating FLLs $[4,5]$. Doppler techniques such as color Doppler imaging (CDI) and power Doppler imaging (PDI) have been used to differentiate hypervascular hepatic tumors, as they can provide information regarding hepatic vascularity patterns [6]. CDI can provide real-time information on vascularity, and PDI can provide improved sensitivity in the detection of blood flow compared with CDI [7]. Unfortunately, however, CDI and PDI have limitations in detecting slow or small vascular flows, which limits the application of Doppler examinations in the evaluation of tumor vascularity and hemodynamics $[8,9]$. In addition to Doppler examinations, contrast-enhanced ultrasonography (CEUS) has additional value for the detection and characterization of FLLS by providing information regarding the detailed vascularity and enhancement pattern $[4,10-13]$. However, CEUS requires intravenous cannulation, additional evaluation time, and an additional expense for contrast agents.

Recently, superb microvascular imaging (SMI) has been developed as a novel Doppler technique that can reduce motion artifacts and preserve high resolution and a high frame rate, improving the detectability of slow or small-vessel flow signals by using specific filters [14]. Several studies have reported that SMI has diagnostic value for characterizing focal lesions in superficial organs, including the thyroid and breast, by revealing slow flow within the lesion $[15,16]$. However, little has been reported on the diagnostic value of SMI in the characterization of FLLS, and these studies had limitations, such as retrospective design or the inclusion of small patient numbers [17-19]. Moreover, as diverse features of hepatic hemangiomas on SMI, which have not been suggested in previously proposed classification [17], have been experienced in the authors' clinical practice, it has become clear that there is a need to investigate a larger number of hepatic hemangiomas and to revise existing classification accordingly.

Therefore, the aim of this study was to investigate and categorize the diverse features of hepatic hemangiomas on SMI with a modified classification in a relatively large prospective study.

\section{Materials and Methods}

\section{Compliance with Ethical Standards}

This prospective study received institutional review board approval of Seoul National University Hospital in Korea (No. 1808-160-967), and all participants gave written informed consent.

\section{Study Population}

From October 2018 to March 2019, consecutive patients who met the eligibility criteria were included in this study. The inclusion criteria were as follows: (1) patients with hemangiomas $(\geq 1 \mathrm{~cm}$ and $<7 \mathrm{~cm}$ ) on US in the right lobe of the liver; (2) patients with contrast-enhanced dynamic computed tomography (CT) and/or magnetic resonance imaging (MRI) prior to the US examination; and (3) patients who gave informed consent. The exclusion criteria were (1) tumor size $<1 \mathrm{~cm}$ or $\geq 7 \mathrm{~cm}$ on US; and (2) a poor sonic window for the evaluation (Fig. 1).

Hemangiomas were diagnosed based on contrast-enhanced CT and/or MRI findings (e.g., peripheral nodular enhancement with progressive centripetal homogeneous filling or immediate homogeneous enhancement in the arterial phase and persistent hyperenhancement in the delayed phase on dynamic CT/MRI that did not change over at least a 2-year follow-up period; and hyperintensity similar to that of cerebrospinal fluid on T2- and heavily $T 2$-weighted images) $[3,20,21]$. Hemangiomas were classified into hemangiomas with a typical enhancing pattern (i.e., typical hemangiomas) and hemangiomas with a rapidly enhancing pattern (i.e., fast-flow hemangiomas) based on the imaging features on dynamic CT/MRI. Typical hemangiomas were defined as lesions with peripheral nodular discontinuous enhancement in the arterial phase, with centripetal progression in the portal and delayed phases, whereas fast-flow hemangiomas were defined as lesions that demonstrated early, uniform, and intense homogeneous enhancement in the arterial phase, with persistent enhancement in the portal and delayed phases [20].

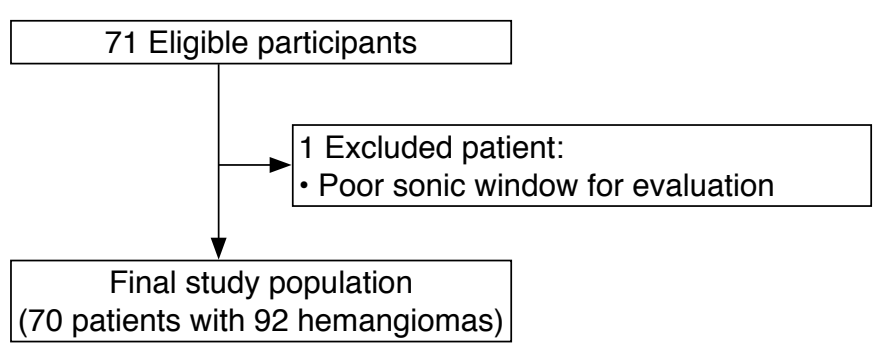

Fig. 1. Flowchart of the study population. 


\section{US Examination and Imaging Analysis}

All US examinations were performed by one of two radiologists (J.Y.L. and S.K.J. with 22 and 5 years of experience in liver US, and with 5 years of experience and initial experience in SMI, respectively) using a diagnostic ultrasound system (Aplio i800, Canon Medical Systems, Tochigi, Japan) with a 1-8 MHz convex probe in patients fasting for at least 6 hours. Initially, the operator scanned the liver with B-mode US and identified the presumed hemangioma by referencing patients' previous CT or MRI scans. When the lesion was not well visualized due to isoechogenicity, the US fusion system using B-mode US and patients' previous CT or MRI scans was used to localize the tumor. Once detected, the size, skin-to-lesion distance (in centimeters), and echogenicity on B-mode US (hyperechoic, isoechoic, or hypoechoic) of the hemangioma were assessed. Thereafter, the US examination in SMI mode was performed on the identified hepatic hemangiomas. If the patient had multiple hemangiomas, SMI features were evaluated by examining the two largest lesions that satisfied the inclusion criteria.

For the SMI-mode US examination, the following parameter settings were used: color velocity scale for SMI mode of 1.0-2.0 $\mathrm{cm} / \mathrm{s}$, color frequency of $5-7 \mathrm{MHz}$, and gain setting adjusted to show optimal imaging. In addition, the vascular information was enhanced by adjusting the temporal smoothing. During the SMI examinations, the best efforts to detect any vascular structures were made, and the imaging data were stored as a video. If any vascularity within the tumor was detected during the SMI examinations, the operator classified the SMI features of the lesion as one of the following patterns: (1) the strip rim pattern, where a few curved vessels encircled the lesion; (2) the nodular rim pattern, which consisted of both curved vessels encircling the lesion and spotty dot-like engorged vessels; (3) the peripheral dot-like pattern, where a few spotty dot-like engorged vessels were visualized at the peripheral area of the lesion; (4) the central dot-like pattern, where a few spotty dot-like engorged vessels were visualized at the central portion of the lesion; (5) the nonspecific vascular pattern, which consisted of a heterogeneous distribution of small vascular clusters without a specific pattern; and (6) the staining pattern, where echogenicity of the lesion was homogeneously increased (Fig. 2) $[17,18]$. After the SMI examination, the SMI features of the lesion were also analyzed by another radiologist, who did not perform the US examination of that lesion and was blinded to the result of the operator, using the stored imaging data to evaluate the interobserver agreement. The results of the operator who performed the SMI examination were used as the representative findings for each lesion for analysis in this study.

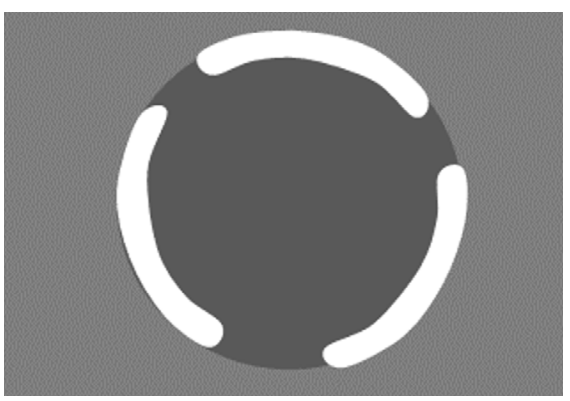

A

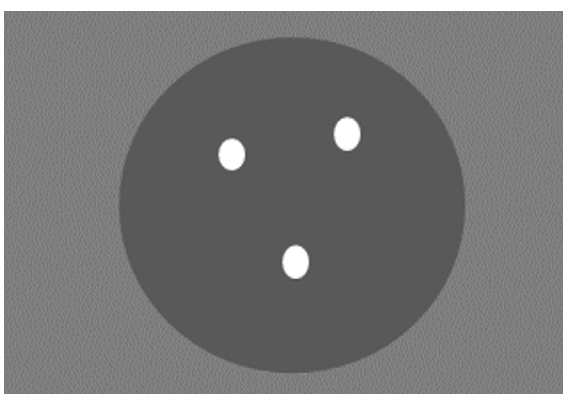

D

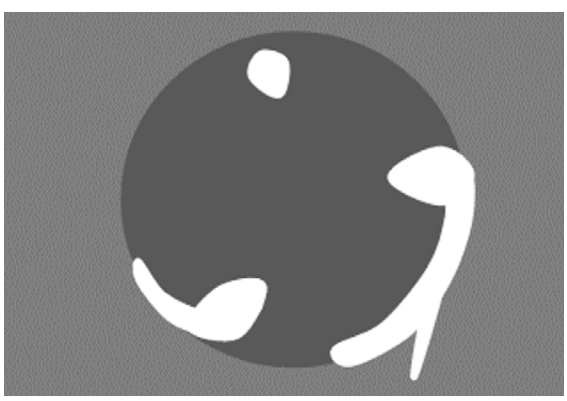

B

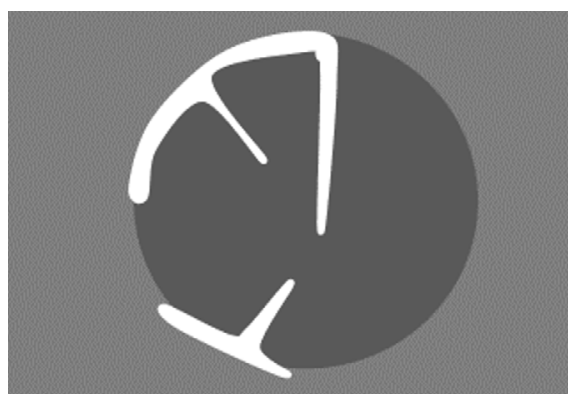

E

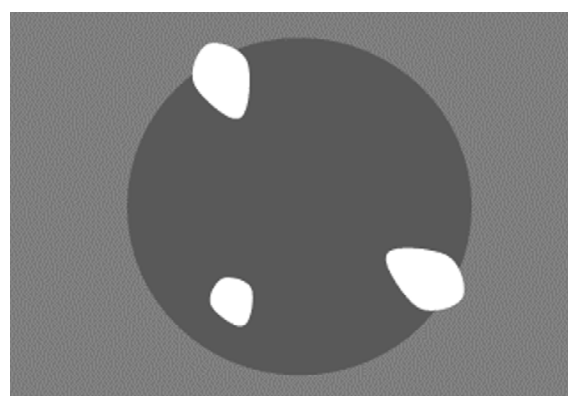

C

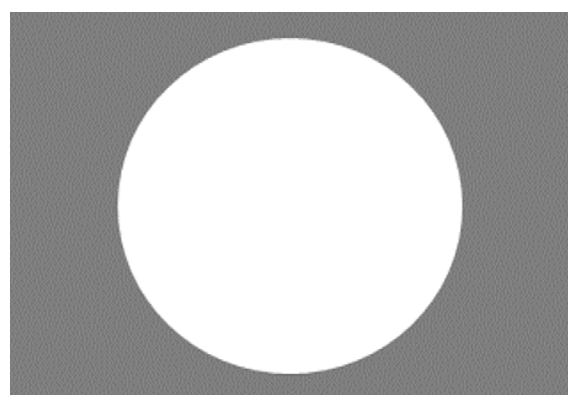

$\mathrm{F}$

Fig. 2. A classification of the superb microvascular imaging features.

A diagram of the superb microvascular imaging features of hepatic nodules is shown: strip rim pattern (A), nodular rim pattern (B), peripheral dot-like pattern (C), central dot-like pattern (D), nonspecific vascular pattern (E), and staining pattern (F). 


\section{Statistical Analysis}

The presence of the SMI signal and frequencies of the SMI features of hemangiomas according to size $(<2 \mathrm{~cm}, \geq 2 \mathrm{~cm})$ [22,23], skin-tolesion distance, echogenicity on B-mode US (hyperechoic, isoechoic, and hypoechoic), and enhancement pattern on dynamic $\mathrm{CT}$ or MRI (typical enhancing pattern or rapidly enhancing pattern [20]) were compared using the Fisher exact test or the chi-square test, as appropriate. The interobserver agreement for the classification of SMI features was evaluated using the Cohen kappa ( $\mathrm{k}$ ) statistic as follows: $k<0.01$, poor agreement; $k=0.01-0.20$, slight agreement; $\mathrm{K}=0.21-0.40$, fair agreement; $\mathrm{K}=0.41-0.60$, moderate agreement; $\mathrm{K}=0.61-0.80$, substantial agreement; and $\mathrm{K}=0.81-0.99$, almost perfect agreement. Statistical analyses were performed using MedCalc version 16.4.1 (MedCalc Software Ltd., Ostend, Belgium). A P-value of less than 0.05 indicated a statistically significant difference.

\section{Results}

Among 71 patients who met the eligibility criteria, one patient was excluded due to a poor sonic window for the evaluation. Finally, a total of 92 lesions (mean size \pm standard deviation, $2.2 \pm 1.2 \mathrm{~cm}$; range, 1 to $7 \mathrm{~cm}$ ) in 70 patients were evaluated (Fig. 1). As 22 patients had multiple hemangiomas, the largest two lesions per patient were evaluated in those 22 patients. Among the 92 lesions, 15 lesions showed a rapidly enhancing pattern, and the remaining 77 lesions showed a typical peripheral globular enhancing pattern with centripetal enhancement on dynamic CT or MRI.

In $67.4 \%$ (62/92) of the hepatic hemangiomas, SMI technology could detect vascularity within the tumor, while 30 of 92 hemangiomas $(32.6 \%, 30 / 92)$ did not show any signal during the $\mathrm{SMI}$ examination. The absence of an SMI signal was not shown in rapidly enhancing hemangiomas ( $0 \%$ [0/30], $\mathrm{P}=0.002)$, and was more frequent in lesions $<2 \mathrm{~cm}$ than in lesions $\geq 2 \mathrm{~cm}$ (44.0\% [22/50] vs. $2.7 \%[8 / 42], P=0.011)$ and in deeply located lesions than in superficially located lesions (skin-to-lesion distance, $5.3 \pm 1.8 \mathrm{~cm}$ vs. $4.3 \pm 2.2 \mathrm{~cm}, \mathrm{P}=0.036$ ).

The specific SMI features of the 62 hepatic hemangiomas are summarized in Table 1. Among hepatic hemangiomas in which vascularity was detected, the strip rim pattern was the most common SMI pattern of hepatic hemangiomas (48.4\%, 30/62) (Fig. $3)$, followed by the nodular rim pattern of spotty dot-like engorged vessels $(37.1 \%, 23 / 62)$ (Fig. 4). Four hemangiomas $(6.5 \%, 4 / 62)$ exhibited a nonspecific vascular pattern, and all of those lesions were larger than or equal to $2 \mathrm{~cm}$. Three hemangiomas exhibited peripheral dot-like patterns $(4.8 \%, 3 / 62)$, and two hemangiomas showed central dot-like patterns $(3.2 \%, 2 / 62)$. Lesion size ( $P=0.365)$, echogenicity on $\mathrm{B}$-mode US ( $\mathrm{P}=0.087)$, and imaging appearance on dynamic $C T$ or MRI $(P=0.451)$ did not show significant differences in SMI features.

The interobserver agreement for classification of SMI features between the two operators showed almost perfect agreement ( $\mathrm{K}$ $=0.852 ; 95 \%$ confidence interval, 0.766 to 0.938 ).

\section{Discussion}

In this study, we suggested six SMI types to demonstrate the vessel distributions and morphology of 92 hepatic hemangiomas. Using SMI technology, we detected vascularity within $67.4 \%$ of hepatic hemangiomas, and the most common SMI feature of hepatic

Table 1. Superb microvascular imaging features of hepatic hemangiomas

\begin{tabular}{|c|c|c|c|c|c|c|c|}
\hline Characteristic & Strip rim & Nodular rim & Peripheral dot-like & Central dot-like & Nonspecific & Staining & P-value \\
\hline Total $(n=62)$ & $30(48.4)$ & $23(37.1)$ & $3(4.8)$ & $2(3.2)$ & $4(6.5)$ & 0 & \\
\hline Size $(\mathrm{cm})$ & & & & & & & 0.365 \\
\hline$\geq 2(n=34)$ & $14(41.2)$ & $13(38.2)$ & $2(5.9)$ & $1(2.9)$ & $4(11.8)$ & 0 & \\
\hline Echogenicity on B-mode US & & & & & & & 0.087 \\
\hline Isoechoic $(n=16)$ & $6(37.5)$ & $7(43.8)$ & 0 & 0 & $3(18.8)$ & 0 & \\
\hline Hypoechoic $(n=14)$ & $5(35.7)$ & $7(50.0)$ & $2(14.3)$ & 0 & 0 & 0 & \\
\hline Imaging appearance on dynamic CT or MRI & & & & & & & 0.451 \\
\hline Rapidly enhancing pattern $(n=15)$ & $7(46.7)$ & $5(33.3)$ & $2(13.3)$ & $0(0)$ & $1(6.7)$ & 0 & \\
\hline Typical enhancing pattern ( $n=47)$ & $23(48.9)$ & $18(38.3)$ & $1(2.1)$ & $2(4.3)$ & $3(6.4)$ & 0 & \\
\hline
\end{tabular}

The data are number of lesions with percentages in parentheses (percentages may not add up to $100 \%$ because of rounding).

US, ultrasonography; CT, computed tomography; MRI, magnetic resonance imaging. 


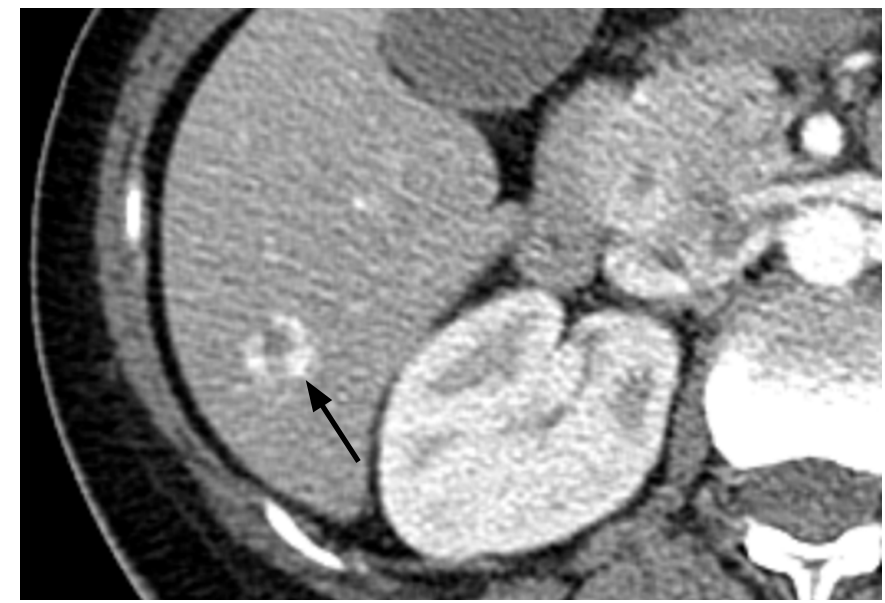

A

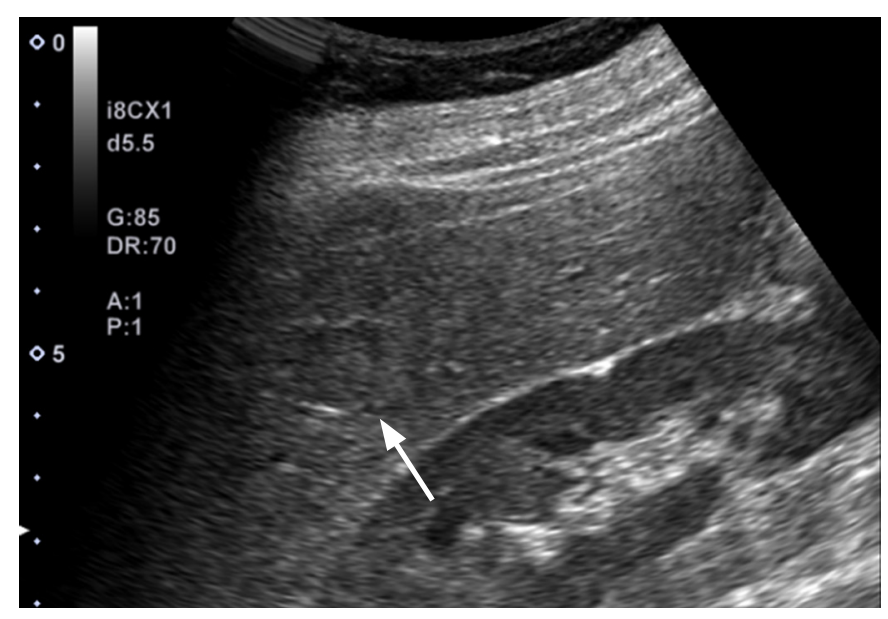

C

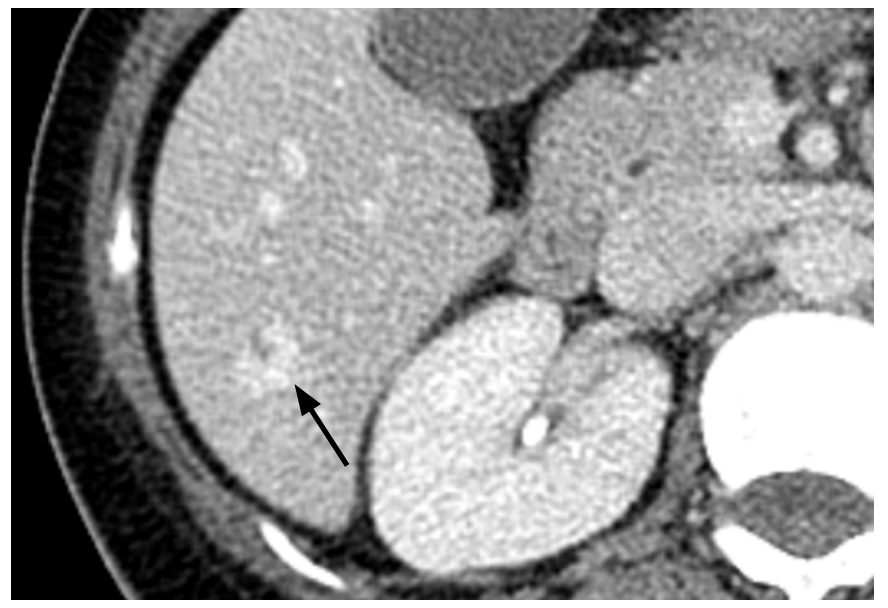

B

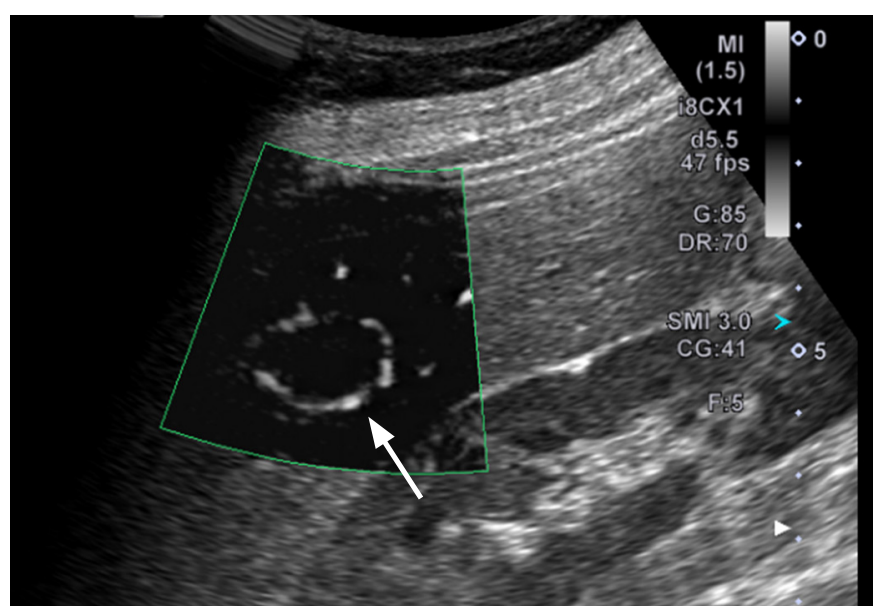

D

Fig. 3. A 2.2-cm hemangioma in a 47-year-old woman.

A, B. On contrast-enhanced computed tomography, a nodular lesion (arrow) exhibits peripheral globular enhancement in the arterial phase (A) and centripetal fill-in in the delayed phase (B), consistent with a hepatic hemangioma. C. B-mode ultrasonography reveals a 2.2-cm isoechoic nodular lesion with a hyperechoic rim (arrow). D. Superb microvascular imaging reveals a well-visualized strip rim pattern of vascularity (arrow).

hemangiomas was the strip rim pattern (48.4\%), followed by the nodular rim pattern (37.1\%), with almost perfect interobserver agreement.

Previous studies have reported the detection of tumoral vascularity and analysis of vascular structures to provide important clues for the differential diagnosis of FLLs $[17,18]$. SMI technology can demonstrate slow or small vascular flow in tumors using a specific filtering technology without the use of a contrast agent, and in our study, the application of SMI technology for the evaluation of hepatic hemangiomas was found to be feasible, as vascularity was demonstrated at the margin or within the lesion in $67.4 \%$ of hepatic hemangiomas. However, $32.6 \%$ of hepatic hemangiomas did not show vascularity using the SMI technique in our study. In addition, the absence of SMI signals was more frequent in small hemangiomas $(<2 \mathrm{~cm})$, hemangiomas with a deeper location, and hemangiomas with a typical enhancement pattern on dynamic CT or MRI. Although the mechanism to explain this result is still unclear, the association between the presence of the SMI signal and dynamic enhancement could be explained by the histologic structure of hepatic hemangioma. According to Yamashita et al. [24], the hemodynamics of hemangiomas depend on the size of the vascular spaces, and rapidly enhancing hemangiomas usually have small vascular spaces, which could be associated with fast vascular flow that is rapid enough to be detected by SMI technology. To evaluate lesions that do not show a signal on SMI, additional US examinations such as CEUS could be helpful.

Moreover, approximately $85 \%$ of hemangiomas that showed vascularity on SMI technology demonstrated either the strip 


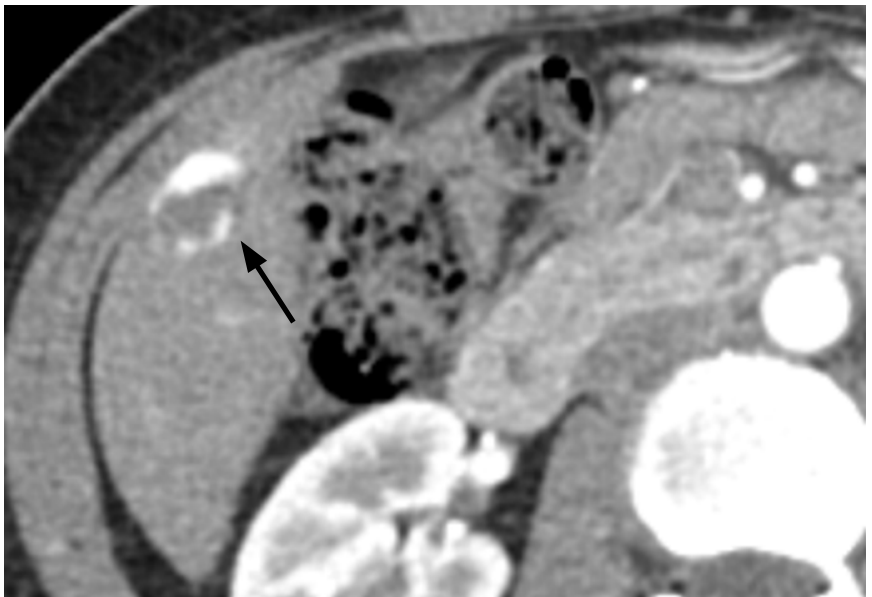

A

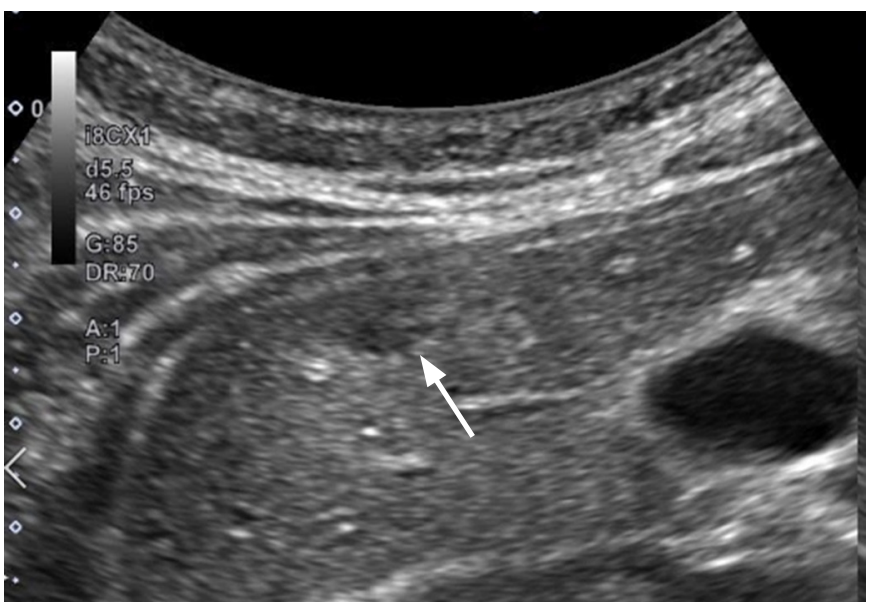

C

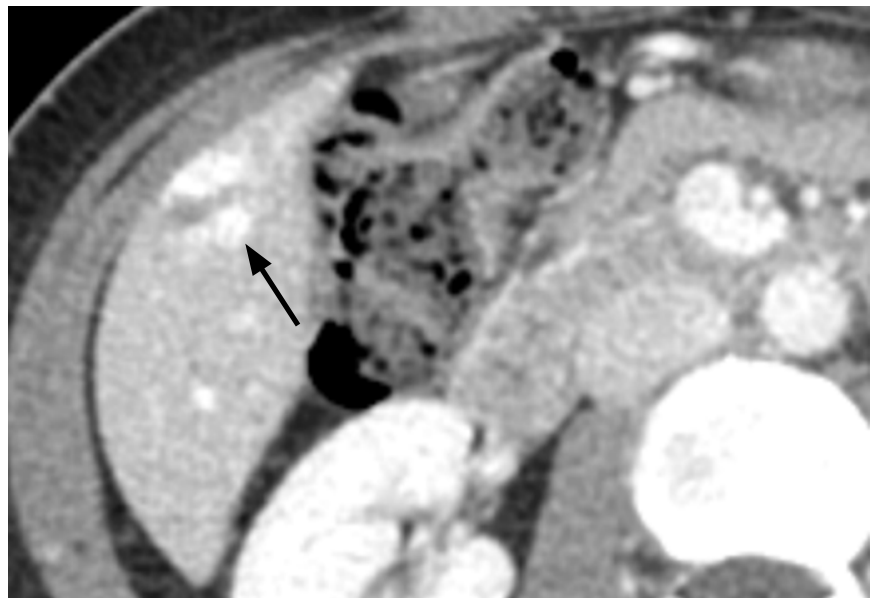

B

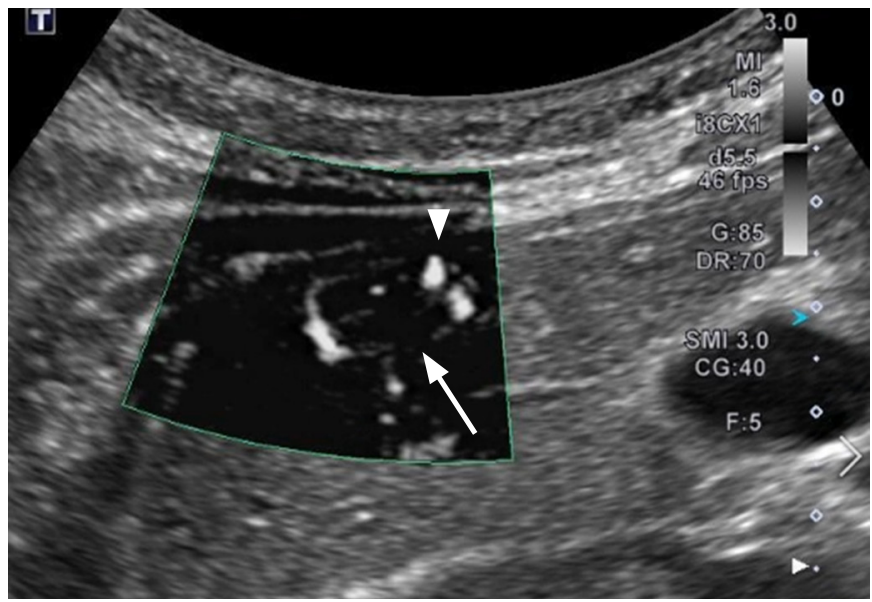

D

Fig. 4. A 1.8-cm hemangioma in a 35-year-old woman.

A, B. Contrast-enhanced computed tomography reveals a $1.8-\mathrm{cm}$ nodule (arrow) with peripheral globular enhancement in the arterial phase (A) and centripetal fill-in in the delayed phase (B), which suggests a hepatic hemangioma. C. B-mode ultrasonography reveals a 1.8$\mathrm{cm}$ hypoechoic nodule with a hyperechoic rim (arrow). D. On superb microvascular imaging, the lesion shows the nodular rim pattern of vascularity (arrow), which is defined as a prominent nodular dot-like structure (arrowhead) with strip rim vascularity.

rim pattern or nodular rim pattern, which is consistent with the findings of previous studies $[17,18]$. In a previous study by Lee et al. [17], 90\% of hepatic hemangiomas showed either a nodular rim pattern or a spotty dot-like pattern. A previous study by He et al. [18] also reported that a substantial proportion (64.7\%) of hemangiomas showed either the strip rim type or nodular rim type. These results could be explained by the pathological features of hepatic hemangiomas. Pathologically, hepatic hemangiomas consist of dilated, large vascular spaces lined by a single row of epithelial cells supported by fibrous septa. The fibrous septa tend to begin centrally, and small vessels communicate with the dilated space at the periphery of the tumor $[25,26]$. Due to fibrous obliteration of the center of the lesions, the vascular space of hepatic hemangiomas usually tends to be arranged in rings or appear C-shaped on angiography, which is consistent with the strip rim pattern of the SMI features of hepatic hemangiomas in our study. In addition, on hepatic arteriography reported in a previous study, $85 \%$ of hepatic hemangiomas showed punctate or nodular contrast accumulation at the arterial phase, and $18 \%$ of hepatic hemangiomas showed early contrast filling in perilesional small portal branches, suggesting drainage vessels, which are correlated with the nodular rim pattern with tangled vessels in our study [27].

There are several limitations in our study. First, the lesions were not histopathologically diagnosed. However, as the examined hemangiomas exhibited typical imaging features on dynamic CT and/or MRI and were stable for at least 2 years of follow-up, 
the likelihood of malignancy was negligible. Second, we did not compare the diagnostic performance of SMI for the detection of tumoral vascularity with that of CDI or PDI. Third, although our study included large number of hepatic hemangiomas, some atypical hemangiomas such as sclerosed/hyalinized hemangiomas (i.e., no enhancement or lack of early enhancement with slight peripheral enhancement in the delayed phase [20]) or hemangiomas larger than $7 \mathrm{~cm}$ were excluded, which limited the generalizability of our study. Fourth, we only evaluated hepatic hemangiomas, not other FLLs, including hepatocellular carcinoma, liver metastasis, and focal nodular hyperplasia, which should be differentiated from hemangiomas. Although in our study, a substantial portion of hepatic hemangiomas showed specific SMI features, there is still a potential overlap with the SMI patterns of other hepatic tumors. Therefore, further studies with a large population including various hepatic lesions are warranted.

In conclusion, the evaluation of the inner vascularity of hepatic hemangiomas with the SMI technique was feasible in most hemangiomas, especially in larger $(\geq 2 \mathrm{~cm})$ or rapidly enhancing hemangiomas. The most frequent SMI patterns of hepatic hemangiomas were the strip rim pattern and the nodular rim pattern.

ORCID: Sun Kyung Jeon: https://orcid.org/0000-0002-8991-3986; Jae Young Lee: https://orcid.org/0000-0001-6946-6042; Joon Koo Han: https://orcid.org/0000-00015916-5545

\section{Author Contributions}

Conceptualization: Lee JY, Han JK. Data acquisition: Jeon SK, Lee JY. Data analysis or interpretation: Jeon SK, Lee JY. Drafting of the manuscript: Jeon SK. Critical revision of the manuscript: Lee JY, Han JK. Approval of the final version of the manuscript: all authors.

\section{Conflict of Interest}

No potential conflict of interest relevant to this article was reported.

\section{References}

1. Mittelstaedt CA. Ultrasound as a useful imaging modality for tumor detection and staging. Cancer Res 1980;40:3072-3078.

2. Doo KW, Lee CH, Choi JW, Lee J, Kim KA, Park CM. "Pseudo washout" sign in high-flow hepatic hemangioma on gadoxetic acid contrast-enhanced MRI mimicking hypervascular tumor. AJR Am J Roentgenol 2009;193:W490-W496.

3. Bajenaru N, Balaban V, Savulescu F, Campeanu I, Patrascu T. Hepatic hemangioma: review. J Med Life 2015;8 Spec Issue:4-11.

4. D'Onofrio M, Crosara S, De Robertis R, Canestrini S, Mucelli RP.
Contrast-enhanced ultrasound of focal liver lesions. AJR Am J Roentgenol 2015;205:W56-W66.

5. Gaiani S, Volpe L, Piscaglia F, Bolondi L. Vascularity of liver tumours and recent advances in doppler ultrasound. J Hepatol 2001;34:474482.

6. Numata K, Tanaka K, Kiba T, Morimoto M, Arata S, Kondo M, et al. Use of hepatic tumor index on color Doppler sonography for differentiating large hepatic tumors. AJR Am J Roentgenol 1997;168:991-995.

7. Choi BI, Kim TK, Han JK, Chung JW, Park JH, Han MC. Power versus conventional color Doppler sonography: comparison in the depiction of vasculature in liver tumors. Radiology 1996;200:5558.

8. Foley WD, Erickson SJ. Color Doppler flow imaging. AJR Am J Roentgenol 1991;156:3-13.

9. Rubin JM, Bude RO, Carson PL, Bree RL, Adler RS. Power Doppler US: a potentially useful alternative to mean frequency-based color Doppler US. Radiology 1994;190:853-856.

10. Lee JY, Minami Y, Choi BI, Lee WJ, Chou YH, Jeong WK, et al. The AFSUMB consensus statements and recommendations for the clinical practice of contrast-enhanced ultrasound using sonazoid. Ultrasonography 2020;39:191-220.

11. Ham T, Jeon JH, Roh Y, Lee $S$, Lee $S$, Kwon H, et al. A novel method to determine hepatic segments using Sonazoid, an ultrasound contrast agent. Ultrasonography 2020;39:94-101.

12. Yang HK, Burns $P N$, Jang HJ, Kono $Y$, Khalili $K$, Wilson SR, et al. Contrast-enhanced ultrasound approach to the diagnosis of focal liver lesions: the importance of washout. Ultrasonography 2019;38:289-301.

13. Bartolotta TV, Taibbi A, Midiri M, Lagalla R. Contrast-enhanced ultrasound of hepatocellular carcinoma: where do we stand? Ultrasonography 2019;38:200-214.

14. Park AY, Seo BK. Up-to-date Doppler techniques for breast tumor vascularity: superb microvascular imaging and contrast-enhanced ultrasound. Ultrasonography 2018;37:98-106.

15. Ma Y, Li G, Li J, Ren WD. The diagnostic value of superb microvascular imaging (SMI) in detecting blood flow signals of breast lesions: a preliminary study comparing SMI to color Doppler flow imaging. Medicine (Baltimore) 2015;94:e1502.

16. Machado P, Segal S, Lyshchik A, Forsberg F. A novel microvascular flow technique: initial results in thyroids. Ultrasound Q 2016;32:6774.

17. Lee DH, Lee JY, Han JK. Superb microvascular imaging technology for ultrasound examinations: Initial experiences for hepatic tumors. Eur J Radiol 2016;85:2090-2095.

18. He MN, LV K, Jiang YX, Jiang TA. Application of superb microvascular imaging in focal liver lesions. World J Gastroenterol 2017;23:7765-7775.

19. Bae JS, Lee JM, Jeon SK, Jang S. Comparison of MicroFlow 
Imaging with color and power Doppler imaging for detecting and characterizing blood flow signals in hepatocellular carcinoma. Ultrasonography 2020;39:85-93.

20. Mathew RP, Sam M, Raubenheimer M, Patel V, Low G. Hepatic hemangiomas: the various imaging avatars and its mimickers. Radiol Med 2020;125:801-815.

21. Klotz T, Montoriol PF, Da Ines D, Petitcolin V, Joubert-Zakeyh J, Garcier JM. Hepatic haemangioma: common and uncommon imaging features. Diagn Interv Imaging 2013;94:849-859.

22. Assy N, Nasser G, Djibre A, Beniashvili Z, Elias S, Zidan J. Characteristics of common solid liver lesions and recommendations for diagnostic workup. World J Gastroenterol 2009;15:3217-3227.

23. Park SW, Kang CH, Kim JH, Kim CJ, Cheong IJ, Kim BH, et al.
Echogenic rim of hepatic hemangioma on abdominal ultrasound. J Korean Soc Med Ultrasound 2001;19:15-19.

24. Yamashita Y, Ogata I, Urata J, Takahashi M. Cavernous hemangioma of the liver: pathologic correlation with dynamic CT findings. Radiology 1997;203:121-125.

25. Abrams RM, Beranbaum ER, Santos JS, Lipson J. Angiographic features of cavernous hemangioma of liver. Radiology 1969;92:308-312.

26. McLoughlin MJ. Angiography in cavernous hemangioma of the liver. Am J Roentgenol Radium Ther Nucl Med 1971;113:50-55.

27. Yu JS, Kim KW, Park MS, Yoon SW. Hepatic cavernous hemangioma in cirrhotic liver: imaging findings. Korean J Radiol 2000;1:185190. 